University of Wollongong

Research Online

Australian Institute for Innovative Materials -

Papers

Australian Institute for Innovative Materials

$1-1-2016$

\title{
Facile Fabrication of Flexible Microsupercapacitor with High Energy Density
}

Yuqing Liu

University of Wollongong, yl037@uowmail.edu.au

Bo Weng

University of Wollongong, Southwest University, bweng@uow.edu.au

Qun Xu

Zhengzhou University, qunxu@zzu.edu.cn

Yuyang Hou

University of Wollongong, yh879@uowmail.edu.au

Chen Zhao

University of Wollongong, cz995@uowmail.edu.au

See next page for additional authors

Follow this and additional works at: https://ro.uow.edu.au/aiimpapers

Part of the Engineering Commons, and the Physical Sciences and Mathematics Commons

Research Online is the open access institutional repository for the University of Wollongong. For further information contact the UOW Library: research-pubs@uow.edu.au 


\title{
Facile Fabrication of Flexible Microsupercapacitor with High Energy Density
}

\begin{abstract}
Flexible microsupercapacitors (fMSCs) with interdigitated electrode design are a promising platform as a power source or energy storage unit for wearable microelectronics. In this work, a facile fabrication of allsolid-state fMSCs is reported through a combination of simple technologies including bar coating and laser etching, to generate the interdigitated patterned electrodes. These novel fMSC devices made with interdigitated patterned electrodes can deliver outstanding areal capacitance $(84.7 \mathrm{mF} \mathrm{cm}-2$ at $5 \mathrm{mV} \mathrm{s}-1$ and $45.5 \mathrm{mF} \mathrm{cm}-2$ at $200 \mathrm{mV}-1)$, high volumetric capacitance $(14.5 \mathrm{~F} \mathrm{~cm}-3$ at $5 \mathrm{mV} \mathrm{s}-1$ and $7.83 \mathrm{~F}$ cm-3 at $200 \mathrm{mV}-1)$, and high cycling stability (94.3\%, 10000 cycles). In addition, the fabrication method employed allows for facile fabrication of various interconnects between several fMSCs on a single device, which enables for the required voltage and/or current output to be tuned easily prior to device fabrication. These fMSCs can power 20 light-emitting diodes even when bent demonstrating versatility for practical applications.
\end{abstract}

\section{Disciplines}

Engineering | Physical Sciences and Mathematics

\section{Publication Details}

Liu, Y., Weng, B., Xu, Q., Hou, Y., Zhao, C., Beirne, S., Shu, K., Jalili, R., Wallace, G. G., Razal, J. M. \& Chen, J. (2016). Facile Fabrication of Flexible Microsupercapacitor with High Energy Density. Advanced Materials Technologies, 1 (9), 1600166-1-1600166-10.

\section{Authors}

Yuqing Liu, Bo Weng, Qun Xu, Yuyang Hou, Chen Zhao, Stephen T. Beirne, Kewei Shu, Rouhollah Jalili, Gordon G. Wallace, Joselito M. Razal, and Jun Chen 
DOI: $10.1002 /$ ((please add manuscript number))

Article type: Full Paper

\section{Facile Fabrication of Flexible Micro-Supercapacitor with High Energy Density}

Yuqing $\mathrm{Liu}^{1}$, Bo Weng ${ }^{2}$, Qun Xu ${ }^{3}$, Yuyang Hou ${ }^{1}$, Chen Zhao ${ }^{1,4}$, Stephen Beirne ${ }^{1}$, Kewei Shu ${ }^{1}$, Rouhollah Jalili ${ }^{1}$, Gordon G. Wallace ${ }^{1}$, Joselito M. Razal ${ }^{5, *}$, and Jun Chen ${ }^{1, *}$

9

10
Y. Liu, Y. Hou, C. Zhao, Dr. S. Beirne, Dr. R. Jalili, Prof. G. G. Wallace, A/Prof. J. Chen Intelligent Polymer Research Institute and ARC Centre of Excellence for Electromaterials Science

University of Wollongong

Wollongong, NSW 2500, Australia

E-mail: junc@uow.edu.au;

Dr. B. Weng

Chongqing Key Lab for Advanced Materials \& Clean Energies of Technologies

Institute for Clean Energy and Advanced Materials

Southwest University

Beibei, Chongqing, 400715, China.

Prof. Q. Xu

College of Materials Science and Engineering

Zhengzhou University

Zhengzhou, 450052, China

C. Zhao

School of Materials and Energy

Guangdong University of Technology

Guangzhou, 510006, China

A/Prof. J. M. Razal

Institute for Frontier Materials

Deakin University

Geelong, VIC 3216, Australia

E-mail: joselito.razal@deakin.edu.au

Keywords: micro-supercapacitor, laser-etching, flexible, high energy density, graphene 
1 Abstract: Flexible microsupercapacitors (fMSCs) with interdigitated electrode design is a

2 promising platform as a power source or energy storage unit for wearable microelectronics. In

3 this work, we report a facile fabrication of all-solid-state fMSC through a combination of

4 simple technologies including bar-coating and laser etching, to generate the interdigitated

5 patterned electrodes. These novel fMSC devices made with interdigitated patterned electrodes

6 can deliver outstanding areal capacitance $\left(84.7 \mathrm{mF} \mathrm{cm}^{-2}\right.$ at $5 \mathrm{mV} \mathrm{s}^{-1}$ and $45.5 \mathrm{mF} \mathrm{cm}^{-2}$ at 200

$\left.7 \mathrm{mV}^{-1}\right)$, high volumetric capacitance $\left(14.5 \mathrm{~F} \mathrm{~cm}^{-3}\right.$ at $5 \mathrm{mV} \mathrm{s}^{-1}$ and $7.83 \mathrm{~F} \mathrm{~cm}^{-3}$ at $200 \mathrm{mV}^{-1}$ )

8 and high cycling stability $(94.3 \%, 10,000$ cycles). In addition, the fabrication method we

9 employed allows for facile fabrication of various interconnects between several fMSCs on a

10 single device, which enables for the required voltage and/or current output to be tuned easily

11 prior to device fabrication. These fMSCs can power 20 light-emitting diodes even when bent

12 demonstrating versatility for practical applications. 


\section{Introduction}

2 Flexible microsupercapacitors (fMSCs) are being studied extensively as alternative power

3 source for flexible and wearable electronics. They are attractive because of their simple

4 construction and ease of fabrication. However, achieving outstanding performance, which are

5 the combination of high energy density, high power density, fast charging and discharging

6 rates for extended periods ( $>10,000$ cycles) and high stability, is proven to be still a challenge.

$7^{1-4]}$ Specifically, fMSCs that are constructed using a conventional design (i.e. two planar

8 electrodes facing each other separated by electrolyte) display performance that fall short of

9 the requirements of microelectronics. ${ }^{[5-7]}$ To enhance device performance, fMSCs that employ

10 an interdigitated layout of active electrodes have been investigated recently aiming at

11 providing better interaction between electrolyte and electrode materials. This design can

12 generate more ion-accessible surface area between electrolyte and electrode materials, which

13 allows ions in electrolyte to permeate into electrode materials more easily. ${ }^{[8-10]}$ Compared

14 with the conventional sandwiched construction, this new design provides the opportunity for attaining higher energy and power densities from a limited space through the control of the dimensions and arrangement of the interdigitated micro-fingers. This design also allows planar interconnection between several devices without the intricate connections of wires. ${ }^{[11]}$ However, present fMSCs with interdigitated electrode design require careful choice of electrode material and sophisticated fabrication techniques for electrode patterning. ${ }^{[12,13]}$

The energy density of fMSCs, which are evaluated by the areal and volumetric capacitance of the whole device, relies on the quality of electrode materials. ${ }^{[14]}$ Recent efforts have focused on using carbon nanomaterials due to their low-cost, excellent electrical conductivity and long-cycle life (See Summary in Table S1)..$^{[2,9,10,15-17]}$ Among these nanomaterials, single layer graphene shows superior volumetric and areal capacitance $\left(600 \mathrm{~F} \mathrm{~cm}^{-3}\right.$ and $21 \mu \mathrm{F} \mathrm{cm}$, respectively) because of its large surface area $\left(2620 \mathrm{~m}^{2} \mathrm{~g}^{-1}\right)^{[18,19]}$. Other graphene derivatives 
1 used in fMSCs such as graphene quantum dots ${ }^{[15]}$, reduced graphene oxide $(\mathrm{rGO})^{[11,20,21]}$, 2 heteroatom-doped graphene ${ }^{[22]}$ also display high volumetric capacitance (15 to $100 \mathrm{~F} \mathrm{~cm}^{-3}$ ) 3 but low areal capacitance $(80-500 \mu \mathrm{F} \mathrm{cm}$ ) because of the relatively thin electrodes 4 employed $(8-300 \mathrm{~nm})$. It is typically observed for graphene electrodes that volumetric 5 capacitance does not scale with electrode thickness because of the re-stacking of individual 6 graphene sheets and insufficient utilization of their surface area. Other forms of 7 nanostructured carbons like onion-like carbon $^{[2]}$, porous activated carbon ${ }^{[16]}$, rGO-carbon 8 nanotubes(CNTs $)^{[17]}$ and porous graphene ${ }^{[13]}$ also provide high ion-accessible surface area, 9 low packing density, and improved areal capacitances $\left(0.2-13 \mathrm{mF} \mathrm{cm}{ }^{-2}\right)$ but the volumetric 10 capacitance is lower $\left(1-7 \mathrm{~F} \mathrm{~cm}^{-3}\right)$. Some pseudo-capacitive materials like PANi ${ }^{[12,23]}$ and 11 metal oxides ${ }^{[24]}$ have been used to increase volumetric capacitance $\left(>50 \mathrm{mF} \mathrm{cm}^{-2}\right.$ and $>20 \mathrm{~F}$ $12 \mathrm{~cm}^{-3}$, respectively) but the capacity retention and cycling stability are poor ( $\leq 90 \%$ at 1,000 cycles) due to the redox involved during the charging and discharging process. To date, however, it is still a challenge to prepare a sub-micrometer thick fMSC electrode with good ion accessibility and high packing density that can deliver both high areal and volumetric capacitance, and exhibit high capacitance retention for extended cycle.

The scalable fabrication of patterned electrodes is another limitation for fMSCs. Lithography 18 can make elaborate electrode patterns but cost can be very high for mass 19 production. $^{[2,11,12,15,17,20-24]}$ Recently, several groups have used laser printing as a writing tool 20 to directly pattern graphene micro-electrodes on its precursor film (e.g. $\mathrm{GO}^{[13,25]}$ and 21 polyimide sheets ${ }^{[26,27]}$, demonstrating a fast and simple technique for fabricating fMSCs. El22 Kady et. al. described a scalable fabrication of graphene fMSCs by direct laser writing on GO 23 films using a standard LightScribe DVD burner, printing more than 100 MSCs on a single 24 disc in 30 minutes. $^{[13]}$ One challenge in the aforementioned approach is that residual 25 precursors are left in between the micro-electrodes, which affect the electrolyte penetration 
1 from the lateral side of the microelectrodes and result in decreased ion diffusion rate. Recently,

2 laser lithography technology (known as laser-cutting system) was also used as an etching tool

3 to create patterned channels on various kinds of film electrodes $\left(\mathrm{MoS}_{2}{ }^{[28]}, \mathrm{aMP}^{[29]}\right.$ and

$\left.4 \mathrm{MDC}^{[30]}\right)$. It is believed that this technology can be applied to a range of electrode materials

5 and film thicknesses provided that residual precursors (or any unwanted materials) are

6 completely removed to create well-separated microelectrodes. This fiber-laser cutter "etches"

7 unwanted material away from the electrode as opposed to "writing" the electrode to generate

8 any practical MSC electrode design on the substrate. The simplicity of this technology (i.e.

9 computer-generated intricate patterns can be "written" on any materials, which include

10 polymers, nanomaterials and many others, and substrates of complex shapes and structures)

11 can pave the way to rational engineering of novel materials for related wearable electronic

12 devices not limited to energy storage applications.

13 Here, we developed an fMSC with superior electrochemical performance by using a facile laser etching process to achieve an interdigitated pattern on a flexible platform made from rGO and PEDOT/PSS composite. The PEDOT/PSS component was effectively stacked in between graphene sheets forming a densely-packed composite electrode $\left(1.4 \mathrm{~g} \mathrm{~cm}^{-3}\right)$, which made it possible to develop fMSCs with excellent areal $\left(84.7 \mathrm{mF} \mathrm{cm}^{-2}\right.$ at $5 \mathrm{mV} \mathrm{s}^{-1}$ and 45.5 $\mathrm{mF} \mathrm{cm} \mathrm{cm}^{-2}$ at $\left.200 \mathrm{mV}^{-1}\right)$ and volumetric capacitance $\left(14.5 \mathrm{~F} \mathrm{~cm}^{-3}\right.$ at $5 \mathrm{mV} \mathrm{s}^{-1}$ and $7.83 \mathrm{~F} \mathrm{~cm}^{-3}$ at

$\left.19200 \mathrm{mV}^{-1}\right)$, as well as good cycling stability (94.3\% capacitance retention after 10,000 cycles).

20 Up to ten interdigitated electrode patterns can be achieved within just 100 seconds, 21 demonstrating a very fast patterning process. The electrode patterns, dimensions and 22 interconnects can be easily adjusted by varying the laser etching parameters in order to 23 achieve the desired energy, power, current and voltage. Furthermore, a long strip of flexible 24 device with 12 tandem fMSCs was demonstrated to power 20 light emitting diodes (LEDs). 


\subsection{Fabrication and characterization of laser-etched rGO-PEDOT/PSS flexible micro-}

\section{2 supercapacitors (fMSCs)}

3 Figure 1a shows the fabrication process of the all-solid-state fMSCs. Briefly, an rGO-

4 PEDOT/PSS dispersion was bar coated onto an Au sputter-coated PET sheet and then dried.

$5 \mathrm{~A} \mathrm{CO}_{2}$ laser cutter system was used to achieve the desired interdigitated patterns by etching

6 the unwanted components (creating channels) and only leaving the active electrode

7 component intact and adhered onto the substrate. Planar fMSCs were made and tested after

8 receiving an electrolyte overcoat. Compared with photolithography ${ }^{[23,31]}$ and wet-etching

9 methods ${ }^{[12,20,32]}$, the overall fMSC fabrication process is simpler, quicker, and potentially

10 lower in cost. The laser etching parameters are easily adjustable to achieve various micron

11 resolution patterns without post-processing and therefore readily scalable. For example, ten

12 fMSC devices with different pattern dimensions (i.e. increasing number of electrode fingers

$13(N)$, denoted as $\mathrm{fMSC}(N)$ in Figure $1 \mathrm{~b})$ can be readily prepared in just 100 seconds during a single laser etching run on a $5 \mathrm{~cm}$ X $7 \mathrm{~cm}$ substrate (Supporting Information Movie S1).

There are a number of factors that were expected to influence the performance of interdigitated fMSCs, which include not only the composition of the active electrode material,

17 but also the electrode design and dimensions such as the distance between adjacent fingers $(d)$, the finger width $(W)$ and length $(L)$ etc. It has been reported that narrow $d$ results in increased capacitance and rate capability because this design enables simultaneous maximization of active material loading and minimization of ion diffusion pathway between the two oppositely charged electrodes (i.e. anode and cathode) ${ }^{[33]}$ In our case, an interdigitated electrode pattern with the lowest $d$ of $\sim 100 \mu \mathrm{m}$ can be achieved without shorting the circuit (Figure 1c); this resolution is similar to previously reported MSCs (Table S1). ${ }^{[2,26,27,29,33]}$ In addition, a small W of $\sim 400 \mu \mathrm{m}$ can also be observed in the $\mathrm{fMSC}(20)$ pattern, which represents $\sim 83 \%$ utilization 
1 electrode. Table S2 lists detailed dimensions of all investigated patterns. It can be seen that

2 for similar $L$ and $d$, an increasing $N$ corresponds to a narrowing of $W$ but this decreases the

3 available active electrode area.

4 Shown in Figure 2a is the digital image of a fabricated all-solid-state $\mathrm{PMSC}(20)$ device during 5 bending and Figure $2 \mathrm{~b}$ displays how a manually bent $\mathrm{fMSC}(20)$ device maintained its 6 integrity. To prove the bendability endurance of fMSCs, the electrochemical performance of $7 \mathrm{fMSC}(20)$ was tested under flat and various bending conditions. Figure $2 \mathrm{~b}$ shows the $\mathrm{CV}$ 8 curves when the device was bent at various curvatures. It can be seen that there is a negligible 9 change in the $\mathrm{CV}$ curves when the device was repeatedly bent from $\mathrm{L}=1 \mathrm{~cm}$ to $\mathrm{L}=0 \mathrm{~cm}$ for up to 1,000 times indicating the fMSC device's excellent performance durability.

11 Some applications require current or voltage that is higher than what can be delivered by a 12 single supercapacitor device. One way to meet this requirement is to electrically connect these 13 supercapacitors in series, in parallel or a combination of these connections. Here, these 14 interconnects were readily achieved using the same laser etching method used for a single fMSC device. As shown in Figure 3, four $\mathrm{AMSC}(20)$ devices were connected in series, in parallel, and a combination of these two by simply changing the etching circuit to form the desired patterns without additional wires for circuitry. The output current of the parallel configuration increased by a factor of four compared with a single fMSC(20) (Figure 3a), and

19 a widened voltage window of $4 \mathrm{~V}$ can be easily achieved by connecting four fMSCs(20) in series (Figure 3b). A combination of two series and two parallel connections enhanced the 21 voltage range to $2 \mathrm{~V}$ without decreasing the output current (Figure 3c). These results indicate 22 that the laser-formed tandem connections between fMSCs enables good control over 23 operating window and current densities. In addition to the ease of fabrication process, the 24 laser-etched fMSCs show great promise for real world applications. 
1 The above results proved the feasibility of scalable fabrication of single and tandem fMSCs

2 via laser-etching technique; the electrochemical performance of fMSCs needs to be optimized

3 for better application. To improve the device performance, the quality of flexible electrode

4 films plays a significant role.

\section{$5 \quad$ 2.2. Characterization of electrode films}

6 The flexible electrode films were prepared directly from rGO-PEDOT/PSS dispersions with

7 different component ratios. The method we used was different from our previous report where

8 films were prepared by first bar coating GO-PEDOT/PSS dispersion onto a substrate and the

9 resulting film then treated chemically to reduce the GO component into $\mathrm{rGO} \cdot{ }^{[34]} \mathrm{A}$ change in method was found necessary because films made by the previous method could be easily

11 delaminated from the substrate and was prone to flaking off during the laser etching process.

12 In contrast, the new method of making rGO-PEDOT/PSS films (i.e. GO was chemically 13 reduced prior to bar coating) resulted in highly flexible films that can be bent and twisted 14 without breaking, delaminating or flaking off the substrate (Supporting Information Figure S1). The cross-sectional SEM images of the interface between the bar-coated active electrode materials and the PET substrate showed excellent adhesion between the two materials (Supporting Information Figure S2). The SEM images of the film cross-sections showed that rGO sheets were densely stacked reminiscent of rGO papers but with PEDOT/PSS intercalated in between rGO sheets (Figure 4a). It is perceived that the PEDOT/PSS have prevented re-stacking of rGO sheets during the reduction process and the subsequent film

21 formation. Based on SEM observations of the edges of the laser-cut rGO-PEDOT/PSS film, the edges remained open and porous similar to its parent (non-laser-cut) film (Figure 4b). This structure suggests that electrolyte can penetrate from the exposed sides of each electrode

24 finger in addition to the conventional electrolyte access from the basal plane of non-patterned electrodes. 
1 The presence of PEDOT/PSS in the composite dispersion played an important role in 2 preventing rGO from aggregation during its reduction. This effect is similar to previous 3 observations when chemical reduction of GO is carried out in the presence of surfactant 4 stabilizers or polymers. ${ }^{[35-37]}$ PEDOT/PSS, a water dispersible conducting polymer, is also 5 known to have non-covalent $\pi-\pi$ interactions between the aromatic rings and the basal planes 6 of graphene sheets and could serve as stabilizer for rGO sheets. ${ }^{[38]}$ Here we found that when 7 GO sheets were chemically reduced in the presence of PEDOT:PSS, aggregate-free 8 dispersions were observed at GO loadings below 50 wt. \%. Upon dilution of the reduced 9 dispersions, aggregates were present only in composite dispersions containing 67 wt. \% and 80 wt. \% GO loadings (Supporting Information Figure S3). The dispersions with 50 wt. \% 11 loading and below remained homogeneous and stable one month after reduction. It is noted 12 that we have previously confirmed that hypophosphorous acid (HPA, the chemical reductant 13 for GO) had negligible effect on the structure PEDOT/PSS and therefore remained 14 electroactive. ${ }^{[34]}$

The XRD results showed that PEDOT/PSS intercalated between the GO sheets and prevented it from re-stacking. In Figure $\mathrm{S} 4 \mathrm{a}$, distinct peaks at $2 \Theta=9.26^{\circ}$ and $2 \Theta=7.9^{\circ}$ were found in GO and GO-PEDOT/PSS samples. These peaks are related to the (002) diffraction of graphene sheet, from which the interlayer $d$ spacing values have been calculated to be 0.95 $19 \mathrm{~nm}$ and $1.12 \mathrm{~nm}$ according to Bragg's law (Equation S1). ${ }^{[39]}$ This slight increase of interlayer spacing in GO-PEDOT/PSS composite film could be attributed to the intercalation of 21 PEDOT/PSS in between GO layers. ${ }^{[40]}$ After reduction, the specific GO peaks at around $10^{\circ}$ disappeared in both rGO and rGO-PEDOT/PSS samples and broad peaks were observed at 23 around $2 \Theta=23.6^{\circ}$ and $2 \Theta=20.0^{\circ}$, which corresponded to decreased interlayer distance of $240.37 \mathrm{~nm}$ and $0.44 \mathrm{~nm}$, respectively compared with samples before reduction. The higher 25 interlayer spacing of the composite sample than pure rGO sample also proved the good 
1 interaction of PEDOT/PSS with individual graphene sheets and its important role in

2 preventing rGO from aggregation during its reduction. This is consistent with the cross-

3 sectional SEM observations.

4 The roles of PEDOT/PSS extend beyond enhancing the conductivity and flexibility of the 5 composite film and preventing rGO aggregation. It has also been reported to exhibit excellent 6 compatibility with polymer gel electrolytes (like $\mathrm{PVA}_{2} \mathrm{H}_{2} \mathrm{SO}_{4}$ and $\mathrm{PVA}-\mathrm{H}_{3} \mathrm{PO}_{4}$ ) as it 7 facilitates electrolyte penetration into the densely packed rGO sheets. ${ }^{[34]}$ It also increased the 8 packing density of the composite film (from $\sim 1.0 \mathrm{~g} \mathrm{~cm}^{-3}$ to $\sim 1.4 \mathrm{~g} \mathrm{~cm}^{-3}$ ) compared to our 9 previous work ${ }^{[34]}$. These two attributes are essential for achieving high volumetric performance. The density achieved for a thick flexible electrode reported here is comparable 11 with literature reported densities for thin film electrodes with high volumetric capacitance $\left(1.33 \sim 1.67 \mathrm{~g} \mathrm{~cm}^{-3}\right)^{[12,41]}$.

As mentioned in our previous work, the commercially sourced PEDOT/PSS we use only acts as the conductive and flexible matrix to prevent re-stacking or rGO layer and most of the capacitance arises from the rGO component of the composite electrode. Thus, effective reduction of the GO to $\mathrm{rGO}$ component is essential. We verified this reduction by Raman. Pristine PEDOT/PSS pellets, GO, GO-PEDOT/PSS and rGO-PEDOT/PSS dispersions were investigated by Raman spectra and shown in Supporting Information Figure S5. The signature peaks for PEDOT/PSS $\left(437,576,988,1256,1368\right.$, and $\left.1428 \mathrm{~cm}^{-1}\right)$ can be observed in the rGO-PEDOT/PSS sample ${ }^{[42]}$ and the three main peaks of rGO at 1323, 1585 and $2624 \mathrm{~cm}^{-1}$ stand for the D, G, and 2D bands, respectively, can also be found in this composite sample. The $\mathrm{D} / \mathrm{G}$ intensity ratio of the composite samples increased from 1.10 (GO-PEDOT/PSS) to 1.24 (rGO-PEDOT/PSS) after HPA treatment suggesting reduction of defects in GO. ${ }^{[43-48,]}$ In addition, the increased 2D intensity attributed to the recovery of crystallinity also verified the reduction of GO. ${ }^{[47,48]}$ This is also confirmed by XPS spectra of PEDOT-GO and PEDOT- 
1 rGO films (shown in Figure S6). The C 1s XPS spetrum corresponding to carbn atoms

2 indifferent oxygen-functional groups: $\mathrm{C}-\mathrm{O}$ and $\mathrm{C}=\mathrm{O}$ (between $286 \mathrm{eV}$ and $288 \mathrm{eV}$ ) exhibits

3 much smaller intensities of these components in the reduced samples (PEDOT-rGO), clearly

4 indicating the reduction of graphene oxide in the composite film.

5 The enhancement of electrical conductivity and electrochemical performance also confirmed

6 the reduction of GO in the composites. To confirm this, pristine PEDOT/PSS and GO-

7 PEDOT/PSS film (with 33 wt. \% GO loading) were prepared, laser patterned and assembled 8 into all-solid-state $\mathrm{fMSC}(20)$ devices in the same process as rGO-PEDOT/PSS film and $9 \mathrm{fMSC}(20)$ and performance evaluated. After reduction, the electrical conductivity increased

10 from $57.7 \mathrm{~S} \mathrm{~cm}^{-1}$ for GO-PEDOT/PSS to $82.8 \mathrm{~S} \mathrm{~cm}^{-1}$ for rGO-PEDOT/PSS film. The fMSC 11 device (i.e. prepared using the rGO-PEDOT/PSS film with 33 wt.\% GO loading) showed 12 remarkable increase in capacitance compared to GO-PEDOT/PSS counterparts (Supporting 13 Information Figure S7). When compared to neat PEDOT/PSS fMSC(20), the rGOPEDOT/PSS fMSC(20) device with the initial GO loading of only 33 wt.\% can deliver increased capacitance by a factor of two. These results verified the dominant contribution of rGO towards device capacitance.

The composition of rGO and PEDOT/PSS in the composite films was optimized for fMSC(20) devices (with mass loading of $3 \mathrm{mg} \mathrm{cm}^{-2}$ ). We note that pure rGO-based fMSC cannot be prepared due to the poor rGO dispersible ability that cracks and flakes off easily from the substrate when cast into films. Evaluation of various composites of fMSCs revealed

21 that the best electrochemical performance (in terms of capacitance and charge transfer rate) was obtained from the device containing $50 \mathrm{wt} \% \%$ GO loading (Supporting Information Figure S8) and was therefore selected for further study.

\subsection{Evaluation of design parameters on the performance of fMSCs}


1 To evaluate the influence of $W$ on device performance, we investigated the devices with

2 different $W$ (Figure 1b) while other parameters such as $L$ and $d$ were kept constant (Table S2).

3 The film thickness (T) and mass loading of all films were kept at $11.6 \mu \mathrm{m}$ and $2 \mathrm{mg} \mathrm{cm}^{-2}$,

4 respectively. Increasing $N$ (i.e. 4, 8, 12, 20, and 32) corresponded to narrower $W$ (i.e. $2400 \mu \mathrm{m}$, $51150 \mu \mathrm{m}, 733 \mu \mathrm{m}, 400 \mu \mathrm{m}$ and $212 \mu \mathrm{m}$, respectively). As illustrated in Figure S9 and Table

6 S2, the loading of active materials decreased from $95.9 \%$ to $73.8 \%$ on each sample when the 7 number of fingers increased from 4 to 32 (based on the same total surface of $1 \mathrm{~cm}^{2}$ ).

8 The device performance was evaluated using cyclic voltammetry (CV) measurements, which 9 was carried out as a function of scan rate $\left(5 \mathrm{mV} \mathrm{s}^{-1}\right.$ to $\left.2000 \mathrm{mV} \mathrm{s}^{-1}\right)$ and a voltage window of $1 \mathrm{~V}$ (Figure 5). At a slow scan rate of $5 \mathrm{mV} \mathrm{s}^{-1}$, all devices exhibited near-perfect rectangular 11 shaped CVs, indicating excellent ion accessibility through the rGO-PEDOT/PSS electrode with only minor ion transport limitation. The capacitance of the whole device (derived from 13 the area of CV curves) increased consistently with the amount of active material in each sample. However, at fast scan rates of 500 and $1000 \mathrm{mV} \mathrm{s}^{-1}, \mathrm{fMSC}(12), \mathrm{fMSC}(8)$ and fMSC(4) samples showed distortion from rectangularity of their CV curves. Only the fMSC(20) and fMSC(32) samples have maintained their rectangular CV shape at these fast scan rates, which indicated that fast ion transport was not maintained when $W$ is $>400 \mu \mathrm{m}$. These results also suggested that the $\mathrm{fMSC}(20)$ sample is superior among the samples investigated because it has the highest capacitance at fast scan rates (excellent rate capability) and the highest active material loading.

21 The observed trend for the scan rate dependence of device capacitance on $W$ was also true for specific capacitance when we normalize the capacitance by volume (Figure 5d) and area (Supporting Information Figure S10a). For example, at the lowest scan rate of $5 \mathrm{mV} \mathrm{s}^{-1}$, the sample with the largest $W$ (fMSC(4) exhibited the largest volumetric and aerial capacitance 25 (23.0 $\mathrm{F} \mathrm{cm}^{-3}$ and $26.7 \mathrm{mF} \mathrm{cm}^{-2}$, respectively). These values decreased much faster than that of 
1 samples with narrow $W$ when scan rate increased. $\mathrm{fMSC}(20)$ exhibited the largest volumetric

2 capacitance among all samples with values of $14.2 \mathrm{~F} \mathrm{~cm}^{-3}$ at $200 \mathrm{mV} \mathrm{s}^{-1}, 12.2 \mathrm{~F} \mathrm{~cm}^{-3}$ at 500

$3 \mathrm{mV} \mathrm{s}^{-1}, 10.0 \mathrm{~F} \mathrm{~cm}^{-3}$ at $1 \mathrm{~V} \mathrm{~s}^{-1}$ and $6.9 \mathrm{~F} \mathrm{~cm}^{-3}$ at $2 \mathrm{~V} \mathrm{~s}^{-1}$. These values surpass that of carbon-

4 based MSCs with sub-micrometer thickness ${ }^{[2,13,26,27,29,30,49,50]}$ and comparable with that of

5 ultrathin-film MSCs ${ }^{[15,20,22]}$ and some pseudo-MSCs ${ }^{[23]}$ (see Table S1 for comparison of

6 literature reported values to this work). The areal capacitance of $\mathrm{fMSC}(20)$ at high scan rates

7 was also superior to all samples. The excellent performance of $\mathrm{fMSC}(20)$ is also reflected in

8 the energy and power densities (based on the volume of the whole device and CV data).

9 fMSC(20) can deliver the highest power density and store comparable energy density

10 compared to other devices (Supporting Information Figure S10b).

11 The device performance was further probed using electrochemical impedance studies. The

12 low frequency part of the impedance spectra of all fMSC samples showed vertical line that rapidly increased, which is characteristic of capacitive behavior (Supporting Information Figure S11). The high frequency domain (Supporting Information Figure S11 inset) showed both small equivalent series resistance (derived from the intercept of the real part of impedance with the $x$-axis) and charge transport resistance (defined by the size of the semicircle at high frequency domain) that decreased with $W$. These results further confirm that the decreasing the ionic diffusion path (through the narrowing of $W$ ) can lower the electrolyte resistance between microelectrodes and thereby increase the ion diffusion rate.

Further characterization of the $\mathrm{fMSC}(20)$ device by galvanostatic charge/discharge (GCD) at various current densities $\left(0.1 \mathrm{~mA} \mathrm{~cm}^{-2}\right.$ to $\left.2 \mathrm{~mA} \mathrm{~cm}^{-2}\right)$ revealed that the slopes of the charge and discharge curves are similar (Figure 6a), indicating excellent capacitive characteristic with very high coulombic efficiency (>95\%). The volumetric capacitances calculated from the GCD curves (inset in Figure 6a) are consistent with those of the CV curves. There was a minimal iR drop observed at the beginning of the current discharge curve, which indicated 
1 low contact resistance in the device. The stability of this device was also evaluated by long

2 term charge-discharge measurements (Figure $6 \mathrm{~b}$ ). The capacitance retention was $85 \%$ when 3 tested for 10,000 cycles at $1 \mathrm{~mA} \mathrm{~cm}{ }^{-2}$. After another 10,000 cycles at $2 \mathrm{~mA} \mathrm{~cm}$, the 4 capacitance remained $84 \%$ of the initial capacitance, suggesting that the device has high 5 stability, long cycle life, and high rate capability. This stability can also be reflected from the 6 nearly $100 \%$ coulombic efficiency for all cycles. Electrochemical impedance was also carried 7 out before and after the long-term cycle tests. The equivalent series resistance (ESR) from the 8 Nyquist plots was only $4.2 \Omega$ (Supporting Information Figure S12), which indicates good 9 electrode conductivity, electrode-current collector contact, and electrolyte conductivity. After these long term cycles, there is only a slight increase in the size of the semi-circle and ESR, 11 suggesting that there is only a small mechanical failure from the polymer doping/dedoping and ion absorbing/desorbing process. ${ }^{[51,52]}$

13 The device performance of $\mathrm{fMSC}(20)$ was compared with a planar supercapacitor prepared 14 from similar electrode/electrolyte composition, thickness and dimensions. This planar supercapacitor was constructed using a conventional sandwiched design (fSSC) where two non-patterned rGO-PEDOT/PSS films $(11.6 \mu \mathrm{m}$-thick, $1 \mathrm{~cm} \times 1 \mathrm{~cm}$ for each film $)$ face each 17 other and are separated by the $\mathrm{PVA} / \mathrm{H}_{3} \mathrm{PO}_{4}$ gel electrolyte. We found a striking difference in performance in favor of the $\mathrm{fMSC}(20)$ as summarized in Supporting Information Figure S13 and Table S3. Without the separator layer, the fMSC device is much thinner $(11.7 \mu \mathrm{m})$ than fSSC $(156 \mu \mathrm{m})$ and can deliver 10 times higher volumetric capacitance than that of the fSSC 21 device (Supporting Information Figure S13a). This result shows the interdigitated design of supercapacitor can supply higher power density for microelectronics than the conventional 23 sandwiched design. It was not straightforward to evaluate the performance of electrode materials from capacitance based on area or volume of device because even when fMSC and fSSC devices were produced from the electrode film of the same size, the dimension of the 
1 assembled device and the active material loading are different (Table S3). Therefore, we also

2 calculated the specific capacitance based on the volume and area of electrode materials based

3 on Equation S6 and S7. As shown in Figure S13c and S13d, fMSC electrodes have slightly

4 higher areal capacitance, volumetric capacitance and rate capability than that of the fSSC

5 electrode. These results show favorable fMSC electrode materials for electrolyte penetration

6 and ion diffusion.

7 The $\mathrm{fMSC}(20)$ device can also deliver high power and energy densities $\left(4.52 \mathrm{~W} \mathrm{~cm}^{-3}\right.$ and 3.05

$8 \mathrm{mWh} \mathrm{cm}^{-3}$, respectively). In comparison with some commercialized Li-ion thin film

9 batteries ${ }^{[2,29]}$ that are presently used in microelectronics, the $\mathrm{fMSC}(20)$ device has comparable

10 energy density, but has power density that is more than three orders of magnitude higher

11 (Figure 6c). Also shown in this figure is the comparison with commercialized supercapacitors

12 where the $\mathrm{fMSC}(20)$ device exhibited significantly higher energy density and comparable

13 power densities. These results demonstrate the exciting potential of these devices for practical

14 applications in energy storage for microelectronics.

\subsection{Increasing the electrode thickness to increase the capacitance of the device}

Electrode thickness is another important parameter that can be used to tune supercapacitor performance. $^{[12,53]}$ In general, device capacitance can be increased by using higher active electrode mass loading, which also results in thicker electrodes ${ }^{[53]}$ However, an increase in the mass loading and electrode thickness can also increase the electrode resistance and affect the overall device performance (rate capability, aerial and volumetric capacitance) ${ }^{[53,39]}$ Here we investigated the device performance of $\mathrm{fMSCs}(20)$ interdigitated device as a function of electrode thickness ( $T$, from $6 \mu \mathrm{m}$ to $58 \mu \mathrm{m}$ ) using $\mathrm{CV}$ measurements. These devices were denoted as $\mathrm{fMSC}(20)-T$ (e.g. $\mathrm{fMSC}(20)-6 \mu \mathrm{m}$ for $\mathrm{fMSCs}$ with electrode thickness of $6 \mu \mathrm{m})$. 
1 At a low scan rate of $5 \mathrm{mV} \mathrm{s}^{-1}$ (Figure 7a), the $\mathrm{CV}$ curves of all devices exhibited excellent

2 rectangular shape and a remarkable increase in the area of these curves for devices with

3 thicker electrode was observed. The linear increase in areal capacitance and slight decrease in

4 volumetric capacitance with increasing electrode thickness (Figure 7c, solid line) indicate that

5 the electrolyte ions had good access to the interior surface of the active materials at this scan

6 rate. With increasing scan rate, the shape of the $\mathrm{CV}$ curves became non-rectangular

7 (Supporting Information Figure S14), as has been reported for thick electrodes due to the

8 reduced ion diffusion time and longer diffusion path. ${ }^{[53]}$ It is noteworthy that for our devices

9 with thick electrodes (fMSC(20)-58 $\mu \mathrm{m})$, the rectangular shape was maintained at a relatively

10 high scan rate of $200 \mathrm{mV} \mathrm{s}^{-1}$ (Figure $7 \mathrm{~b}$ ) indicating that good rate capability was retained.

11 The areal capacitances of both $84.7 \mathrm{mF} \mathrm{cm}^{-2}$ at $5 \mathrm{mV} \mathrm{s}^{-1}$ and $45.5 \mathrm{mF} \mathrm{cm}^{-2}$ at $200 \mathrm{mV} \mathrm{s}^{-1}$

12 achieved by the device of $\mathrm{fMSC}(20)-58 \mu \mathrm{m}$ are significantly higher than the previously 13 reported values for carbon-based MSCs $\left(2.4-12.4 \mathrm{mF} \mathrm{cm}^{-2}\right)^{[13,26,27,29,30]}$ and MSCs with 14 pseudocapacitive materials $\left(1.2-56.3 \mathrm{mF} \mathrm{cm}^{-2}\right)^{[12,23,24]}$ as listed in Table S1. Also, slightly decreased volumetric capacitances of $14.5 \mathrm{~F} \mathrm{~cm}^{-3}$ at $5 \mathrm{mV} \mathrm{s}^{-1}$ and $7.83 \mathrm{~F} \mathrm{~cm}^{-3}$ at $200 \mathrm{mV} \mathrm{s}^{-1}$ are still comparable with carbon-based MSCs with excellent volumetric capacitive behavior as listed in Table S1. ${ }^{[2,13,15,20,26,27,29,30,54]}$ In addition, this device delivers an energy density that 18 is almost four times higher than the $\mathrm{fMSC}(20)-11 \mu \mathrm{m}\left(13.1 \mu \mathrm{Wh} \mathrm{cm}^{-2}\right.$ vs. $3.46 \mu \mathrm{Wh} \mathrm{cm}^{-2}$, respectively). This excellent device performance, which is much higher than literature reported values, was achieved with only a small decrease in power density (from $8.47 \mathrm{~mW}$ $21 \mathrm{~cm}^{-2}$ to $7.51 \mathrm{~cm}^{-2}$, respectively).

The symmetric GCD curves of fMSC (20)-58 $\mu \mathrm{m}$ under different current densities signified good capacitive characteristic. The areal capacitances calculated from the GCD curves (inset in Figure 7e) are consistent with those of the CV curves. Meanwhile, the capacitance retention of $94.3 \%$ after 10,000 charge/discharge cycles at a current density of $1 \mathrm{~mA} \mathrm{~cm}{ }^{-2}$ and the 
1 excellent coulombic efficiency (close to $100 \%$ ) during all 10,000 cycles demonstrate its high

2 cycling stability (Figure $7 \mathrm{~g}$ ).

\section{2.5. Integration of fMSCs}

4 Due to the low energy stored in limited area, there are few demonstrations of single MSCs as 5 power sources for potential application. In our case, the $\mathrm{fMSC}(20)-58 \mu \mathrm{m}$ device with high 6 energy density is powerful enough to power a light-emitting diode (LED) (1V) when fully 7 charged (Figure 8a). As most of applications require higher voltage window, tandem fMSCs 8 connected in 6 series $\times 2$ parallel were fabricated from a long strip of flexible rGO-

9 PEDOT/PSS film $(1.5 \mathrm{~cm} \times 18 \mathrm{~cm})$. This device was rolled around a wrist to demonstrate its 10 robustness while in operation. Using this configuration, this fMSC device successfully 11 powered 20 small LEDs when fully charged to $6 \mathrm{~V}$ (Figure $8 \mathrm{~b}$ and Movie S2). These results 12 highlight the exciting potential of these fMSCs in a wide range of applications, especially 13 wearable electronics.

14

\section{Conclusion}

In Summary, an all-solid-state graphene-PEDOT/PSS-based fMSC have been simply and successfully fabricated through a combination of bar-coating and laser-etching techniques. We demonstrated that the laser-etching is a scalable technique to generate interdigitated electrode patterns due to the simple, quick, and low-cost process. The dimensions and interconnect patterns of these laser-fabricated electrode are easily adjustable to achieve the desired energy, power, current and voltage outputs. In addition, the single fMSC device showed little changes in electrochemical performance when bent at various angles repeatedly for 1,000 times, indicating the robustness required by many practical applications. In the densely-stacked composite electrode, the presence of PEDOT/PSS prevented rGO sheets from re-stacking and improved the electrochemical performance of the assembled fMSCs. After 
1 tuning the micro-electrode design ( $W$ and $T$ ), our all-solid-state $\mathrm{fMSC}(20)$ device made from a

$258 \mu \mathrm{m}$-thick electrode displayed a high areal capacitance $\left(84.7 \mathrm{mF} \mathrm{cm}^{-2}\right.$ at $5 \mathrm{mV} \mathrm{s}^{-1}$ and 45.5

$3 \mathrm{mF} \mathrm{cm}^{-2}$ at $\left.200 \mathrm{mV}^{-1}\right)$, volumetric capacitance $\left(14.5 \mathrm{~F} \mathrm{~cm}^{-3}\right.$ at $5 \mathrm{mV} \mathrm{s}^{-1}$ and $7.83 \mathrm{~F} \mathrm{~cm}^{-3}$ at 200

$\left.4 \mathrm{mV}^{-1}\right)$ and excellent cycling property (>10,000 cycles). The demonstration of powered LED

5 lights using the single and tandem fMSCs highlight the great potential of the scalable fMSCs

6 in real application. Furthermore, the success of scalable fabrication of tandem fMSCs

7 demonstrates that the Laser-Lithography technology we employ has the potential to make

8 practical advances in device engineering, particularly for device fabrication and design of

9 flexible devices requiring intricate connections, shapes and structures.

\section{Experimental Section}

11 Materials: Graphite flakes, diethylene glycol (DEG), poly (vinyl alcohol) (PVA, Mw: 146000

$12 \sim 186000$ ) and hypophosphorous acid (HPA) were purchased from Sigma-Aldrich.

13 Orthophosphoric acid $\left(\mathrm{H}_{3} \mathrm{PO}_{4}, 85 \%\right)$ were obtained from Chem-Supply. Poly (3,4-

14 ethylenedioxythiophene)/poly(styrenesulfonate) (PEDOT/PSS) pellets was Orgacon ${ }^{\mathrm{TM}}$ DRY re-dispersible product from Agfa company.

Preparation of rGO-PEDOT/PSS dispersion: rGO-PEDOT/PSS dispersions were prepared by reducing GO under the presence of PEDOT/PSS in the dispersion (GO-PEDOT/PSS dispersion). Firstly, PEDOT/PSS pellets were added into GO dispersions (prepared by improved Hummers method), ${ }^{[34,55]}$ stirred overnight and then sonicated for 30 minutes (Branson B5500R-DTH bath sonicator). Samples with various GO loadings of 20 wt.\%, 33 wt. $\%, 50$ wt. $\%, 67$ wt. $\%$ and 80 wt. $\%$ were prepared. In each sample, the total concentration (solid content) of PEDOT/PSS and GO was kept at $15 \mathrm{mg} \mathrm{mL}^{-1}$. HPA (reduction agent) was added into the as-prepared GO-PEDOT/PSS dispersion at GO:HPA of 1:20 by weight, stirred at $90{ }^{\circ} \mathrm{C}$ for 5 hours, and then dialyzed for 3 days to remove the residual acid. Diethylene 
1 glycol was added into the obtained rGO-PEDOT/PSS dispersion at a DEG:solid content

2 weight ratio of 1.86:1. Pure rGO dispersion was also prepared using the same method for 3 comparison.

4 Fabrication of rGO-PEDOT/PSS flexible microsupercapacitors (fMSCs): rGO-PEDOT/PSS

5 fMSCs were produced by direct laser cutting on rGO-PEDOT/PSS films as illustrated in

6 Figure 1a. rGO-PEDOT/PSS films were prepared by bar-coating a thin layer of rGO-

7 PEDOT/PSS dispersion on a $100 \mathrm{~nm} \mathrm{Au} \mathrm{sputter} \mathrm{coated} \mathrm{PET} \mathrm{plastic} \mathrm{film} \mathrm{(100} \mu \mathrm{m}$ thickness)

8 to a fixed shape and size. The film's thickness was controlled by the volume of dispersion

9 used per unit area of the film. Films were first dried overnight at room temperature and then heated at $60{ }^{\circ} \mathrm{C}$ in air overnight. The interdigitated finger electrodes were patterned on these

11 films by a $10.6 \mu \mathrm{m} \mathrm{CO}$ laser cutting system (Universal PLS6MW Multi-Wavelength Laser

12 Platform, $30 \mathrm{~W}$, spot size of $\sim 25 \mu \mathrm{m})$ under ambient conditions. Firstly, layers of active

13 materials and Au were selectively etched to form interdigitated patterns as shown in Figure 1.

14 The laser power was set between $5 \%$ to $25 \%$ depending on film composition and thickness and the scan speed fixed at $10 \%$. Interdigitated electrodes were patterned with a length $(l)$ of $8 \mathrm{~mm}$, an interspacing distance $(d)$ of $100 \mu \mathrm{m}$ between two neighboring finger electrodes, and

17 with different widths $(W)$ ranging from $212 \mu \mathrm{m}$ to $2.4 \mathrm{~mm}$ depending on the number of 18 fingers in a total area of $1 \mathrm{~cm} \times 1 \mathrm{~cm}$. Then two square shaped electrical contact pads $(3 \mathrm{~mm}$ $19 \times 3 \mathrm{~mm}$ ) connecting positive and negative electrode were formed by etching only the active materials layer leaving the Au layer.

21 Patterned electrodes were assembled into all-solid-state fMSCs by the following methods. Firstly, sliver paint (Ted Pella) was applied on the contact pads, followed by extending with conductive copper tapes, which were connected to electrochemical workstation during testing.

24 A hot polymer gel electrolyte $\mathrm{PVA}-\mathrm{H}_{3} \mathrm{PO}_{4}$ (heated to $85^{\circ} \mathrm{C}$ prior to use) was then drop cast onto the projected area and solidified in air overnight. The $\mathrm{PVA}-\mathrm{H}_{3} \mathrm{PO}_{4}$ electrolyte was 
1 prepared according to the previously reported method ${ }^{[34]}$. PVA powder $(4 \mathrm{~g}), \mathrm{H}_{3} \mathrm{PO}_{4}(6 \mathrm{~g})$ and

2 deionized water $(40 \mathrm{~mL})$ were mixed together and then heated to around $85{ }^{\circ} \mathrm{C}$ under 3 magnetic stirring until the solution became clear.

4 Characterization and measurements: Physical characterization was achieved by optical 5 microscopy, scanning electron microscopy (SEM), four-probe conductivity, surface 6 profilometer, X-ray diffraction (XRD) and Raman spectroscopy. Patterns of laser-cut micro7 devices were observed by optical microscopy (Leica DM6000 upright microscope). The 8 cross-section morphology of drop-cast and laser-cut films were investigated from a JEOL JSM-7500FA field emission SEM with the accelerating voltage set at $5.0 \mathrm{kV}$ and the emission current at $10 \mathrm{~mA}$. The film thicknesses were measured using a Veeco Dektak 150 surface 11 profilometer and sheet resistances were measured using a Jandel RM3-AR four-probe conductivity. XRD was performed on a GBC MMA XRD $(\lambda=1.54 \AA)$ with the voltage and 13 current kept at $-40 \mathrm{kV}$ and $25 \mathrm{~mA}$, respectively. Raman spectroscopy was carried out on a Jobin-Yvon Horbia 800 using a $632.81 \mathrm{~nm}$ laser. The data analysis was carried out using Labspec V.5.45.09 software. The electrical conductivity of the rGO-PEDOT/PSS film was measured by standard four-point probe conductivity (JANDEL RM3-AR).

Electrochemical performances of as-prepared fMSCs were investigated by cyclic voltammetry 18 (CV), electrochemical impedance spectroscopy (EIS) and galvanostatic charge/discharge 19 techniques. CVs were performed using a CHI720 electrochemical work station. Galvanostatic charge/discharge and long-term cycling tests were carried out with a Neware Galvanostat (100 mA, 5 V) equipped with Test Control V.5.0 software. EIS were obtained using Solartron SI1260 Impedance Analyser and EG\&G Instruments Princeton Applied Research Potentiostat/Galvanostat Model 283 employing a frequency range of $100 \mathrm{kHz}$ to $0.01 \mathrm{~Hz}$ and an $\mathrm{AC}$ amplitude of $10 \mathrm{mV}$ at open circuit potential. All experiments were performed at room 
1 temperature. Calculations of specific capacitance and the energy and power densities are

2 discussed in detail in Supporting Information Information.

\section{Supporting Information}

\section{Acknowledgements}

5 Funding from the Australian Research Council Centre of Excellence Scheme (CE 140100012)

6 is gratefully acknowledged. The authors would like to thank the Australian National 7 Fabrication Facility - Materials node and UOW Electron Microscopy Centre for equipment 8 use. GGW and JMR gratefully acknowledge the ARC for support under the Australian 9 Laureate Fellowship (FL110100196) and Future Fellowship (FT130100380) schemes, respectively. YL would like to acknowledge the support of the CSC scholarship from the

11 Ministry of Education of P. R. China

12

13

14

15

16

17
Received: ((will be filled in by the editorial staff))

Revised: ((will be filled in by the editorial staff)) Published online: ((will be filled in by the editorial staff))

[1] J. M. Miller, Ultracapacitor Applications; IET, 2011.

[2] D. Pech, M. Brunet, H. Durou, P. Huang, V. Mochalin, Y. Gogotsi, P.-L. Taberna, P. Simon, Nat. Nanotechnol. 2010, 5, 651.

[3] X. Lang, A. Hirata, T. Fujita, M. Chen, Nat. Nanotechnol. 2011, 6, 232.

[4] Y. Masaki, J. B. Ralph, K. Akiya, Lithium-Ion Batteries, Science and Technologies; Springer, 2009.

[5] Z. L. Wang, Nano Today 2010, 5, 512.

[6] I. F. Akyildiz, W. Su, Y. Sankarasubramaniam, E. Cayirci, Comput. Netw. 2002, 38, 393.

[7] Z. L. Wang, W. Wu, Angew. Chem. 2012, 124, 11868; Z. L. Wang, W. Wu, Angew. Chem. Int. Ed. 2012, 51, 11700. 
[8] J.-H. Sung, S.-J. Kim, S.-H. Jeong, E.-H. Kim, K.-H. Lee, J. Power Sources 2006, 162, 1467.

[9] J. Chmiola, C. Largeot, P.-L. Taberna, P. Simon, Y. Gogotsi, Science 2010, 328, 480.

[10] P. Huang, M. Heon, D. Pech, M. Brunet, P.-L. Taberna, Y. Gogotsi, S. Lofland, J. D. Hettinger, P. Simon, J. Power Sources 2013, 225, 240.

[11] Z. Niu, L. Zhang, L. Liu, B. Zhu, H. Dong, X. Chen, Adv. Mater. 2013, 25, 4035.

[12] Z. Wu, K. Parvez, S. Li, S. Yang, Z. Liu, S. Liu, X. Feng, K. Müllen, Adv. Mater. 2015, 27, 4054.

[13] M. F. El-Kady, R. B. Kaner, Nat. Commun. 2013, 4, 1475.

[14] M. Beidaghi, Y. Gogotsi, Energy Environ. Sci. 2014, 7, 867.

[15] W.-W. Liu, Y.-Q. Feng, X.-B. Yan, J.-T. Chen, Q.-J. Xue, Adv. Funct. Mater. 2013, 23, 4111.

[16] D. Pech, M. Brunet, P.-L. Taberna, P. Simon, N. Fabre, F. Mesnilgrente, V. Conédéra, H. Durou, J. Power Sources 2010, 195, 1266.

[17] M. Beidaghi, C. Wang, Adv. Funct. Mater. 2012, 22, 4501.

[18] J. Xia, F. Chen, J. Li, N. Tao, Nat. Nanotechnol. 2009, 4, 505.

[19] M. F. El-Kady, V. Strong, S. Dubin, R. B. Kaner, Science 2012, 335, 1326.

[20] Z.-S. Wu, K. Parvez, X. Feng, K. Müllen, Nat. Commun. 2013, 4, 2487.

[21] D. Qi, Z. Liu, Y. Liu, W. R. Leow, B. Zhu, H. Yang, J. Yu, W. Wang, H. Wang, S. Yin, X. Chen, Adv. Mater. 2015, 27, 5559

[22] Z.-S. Wu, K. Parvez, A. Winter, H. Vieker, X. Liu, S. Han, A. Turchanin, X. Feng, K. Müllen, Adv. Mater. 2014, 26, 4552.

[23] K. Wang, W. Zou, B. Quan, A. Yu, H. Wu, P. Jiang, Z. Wei, Adv. Energy Mater. 2011, $1,1068$.

[24] X. Wang, B. D. Myers, J. Yan, G. Shekhawat, V. Dravid, P. S. Lee, Nanoscale 2013, 5, 4119.

[25] W. Gao, N. Singh, L. Song, Z. Liu, A. L. M. Reddy, L. Ci, R. Vajtai, Q. Zhang, B. Wei, P. M. Ajayan, Nat. Nanotechnol. 2011, 6, 496.

[26] J. Lin, Z. Peng, Y. Liu, F. Ruiz-Zepeda, R. Ye, E. L. G. Samuel, M. J. Yacaman, B. I. Yakobson, J. M. Tour, Nat. Commun. 2014, 5, 5714. 
[27] Z. Peng, R. Ye, J. A. Mann, D. Zakhidov, Y. Li, P. R. Smalley, J. Lin, J. M. Tour, ACS Nano 2015, 9, 5868.

[28] L. Cao, S. Yang, W. Gao, Z. Liu, Y. Gong, L. Ma, G. Shi, S. Lei, Y. Zhang, S. Zhang, R. Vajtai, P. M. Ajayan, Small 2013, 9, 2905.

[29] H.-C. Huang, C.-J. Chung, C.-T. Hsieh, P.-L. Kuo, H. Teng, Nano Energy 2016, 21, 90.

[30] P. Yadav Basu, A., Suryawanshi, A., Game, O., Ogale, S., Adv. Mater. Interfaces 2016, 3, 1600057.

[31] N. Kurra, M. K. Hota, H. N. Alshareef, Nano Energy 2015, 13, 500.

[32] Z. Wu, Z. Liu, K. Parvez, X. Feng, K. Müllen, Adv. Mater. 2015, 27, 3669.

[33] W. Liu, C. Lu, X. Wang, R. Y. Tay, K. Tay, ACS Nano 2015, 9, 1528.

[34] Y. Liu, B. Weng, J. M. Razal, Q. Xu, C. Zhao, Y. Hou, S. Seyedin, R. Jalili, G. G. Wallace, J. Chen, Sci. Rep. 2015, 5, 17045.

[35] S.-Z. Zu, B.-H. Han, J. Phys. Chem. C 2009, 113, 13651.

[36] H. Bai, Y. Xu, L. Zhao, C. Li, G. Shi, Chem. Commun. 2009, 13, 1667.

[37] Z.-B. Liu, Y.-F. Xu, X.-Y. Zhang, X.-L. Zhang, Y.-S. Chen, J.-G. Tian, J. Phys. Chem. B 2009, 113, 9681.

[38] L. K. H. Trang, T. Thanh Tung, T. Young Kim, W. S. Yang, H. Kim, K. S. Suh, Polym. Int. 2012, 61, 93.

[39] D. Antiohos, M. S. Romano, J. M. Razal, S. Beirne, P. Aitchison, A. I. Minett, G. G. Wallace, J. Chen, J. Mater. Chem. A 2014, 2, 14835.

[40] K. Zhang, L. L. Zhang, X. S. Zhao, J. Wu, Chem. Mater. 2010, 22, 1392.

[41] M. Li, Z. Tang, M. Leng, J. Xue, Adv. Funct. Mater. 2014, 24, 7495.

[42] X. Zhang, D. Chang, J. Liu, Y. Luo, J. Mater. Chem. 2010, 20, 5080.

[43] S. Stankovich, D. A. Dikin, R. D. Piner, K. A. Kohlhaas, A. Kleinhammes, Y. Jia, Y. $\mathrm{Wu}, \mathrm{S}$. T. Nguyen, R. S. Ruoff, Carbon 2007, 45, 1558.

[44] X. Cao, D. Qi, S. Yin, J. Bu, F. Li, C. F. Goh, S. Zhang, X. Chen, Adv. Mater. 2013, 25, 2957.

[45] C. Gómez-Navarro, R. T. Weitz, A. M. Bittner, M. Scolari, A. Mews, M. Burghard, K. Kern, Nano Lett. 2007, 7, 3499.

[46] Z. Niu, J. Chen, H. H. Hng, J. Ma, X. Chen, Adv. Mater. 2012, 24, 4144. 
1 [47] G. Eda, M. Chhowalla, Adv. Mater. 2010, 22, 2392.

2 [48] J. Chen, R. L. Shepherd, J. M. Razal, X. Huang, W. Zhang, J. Zhao, A. T. Harris, S. Wang, A. I. Minett, H. Zhang, ACS Appl. Mater. Interfaces 2013, 5, 7676.

4 [49] M. Beidaghi, C. Wang, Electrochimica Acta 2011, 56, 9508.

[50] Z. Peng, J. Lin, R. Ye, E. L. G. Samuel, J. M. Tour, ACS Appl. Mater. Interfaces 2015, 7,3414 .

[51] D. Antiohos, K. Pingmuang, M. S. Romano, S. Beirne, T. Romeo, P. Aitchison, A. Minett, G. Wallace, S. Phanichphant, J. Chen, Electrochim. Acta 2013, 101, 99.

[52] D. Antiohos, G. Folkes, P. Sherrell, S. Ashraf, G. G. Wallace, P. Aitchison, A. T. Harris, J. Chen, A. I. Minett, J. Mater. Chem. 2011, 21, 15987.

[53] Z. Su, C. Yang, C. Xu, H. Wu, Z. Zhang, T. Liu, C. Zhang, Q. Yang, B. Li, F. Kang, J. Mater. Chem. A 2013, 1, 12432.

[54] J. Lin, C. Zhang, Z. Yan, Y. Zhu, Z. Peng, R. H. Hauge, D. Natelson, J. M. Tour, Nano Lett. 2013, 13, 72

[55] D. C. Marcano, D. V Kosynkin, J. M. Berlin, A. Sinitskii, Z. Sun, A. Slesarev, L. B. 16 Alemany, W. Lu, J. M. Tour, ACS Nano 2010, 4, 4806. 

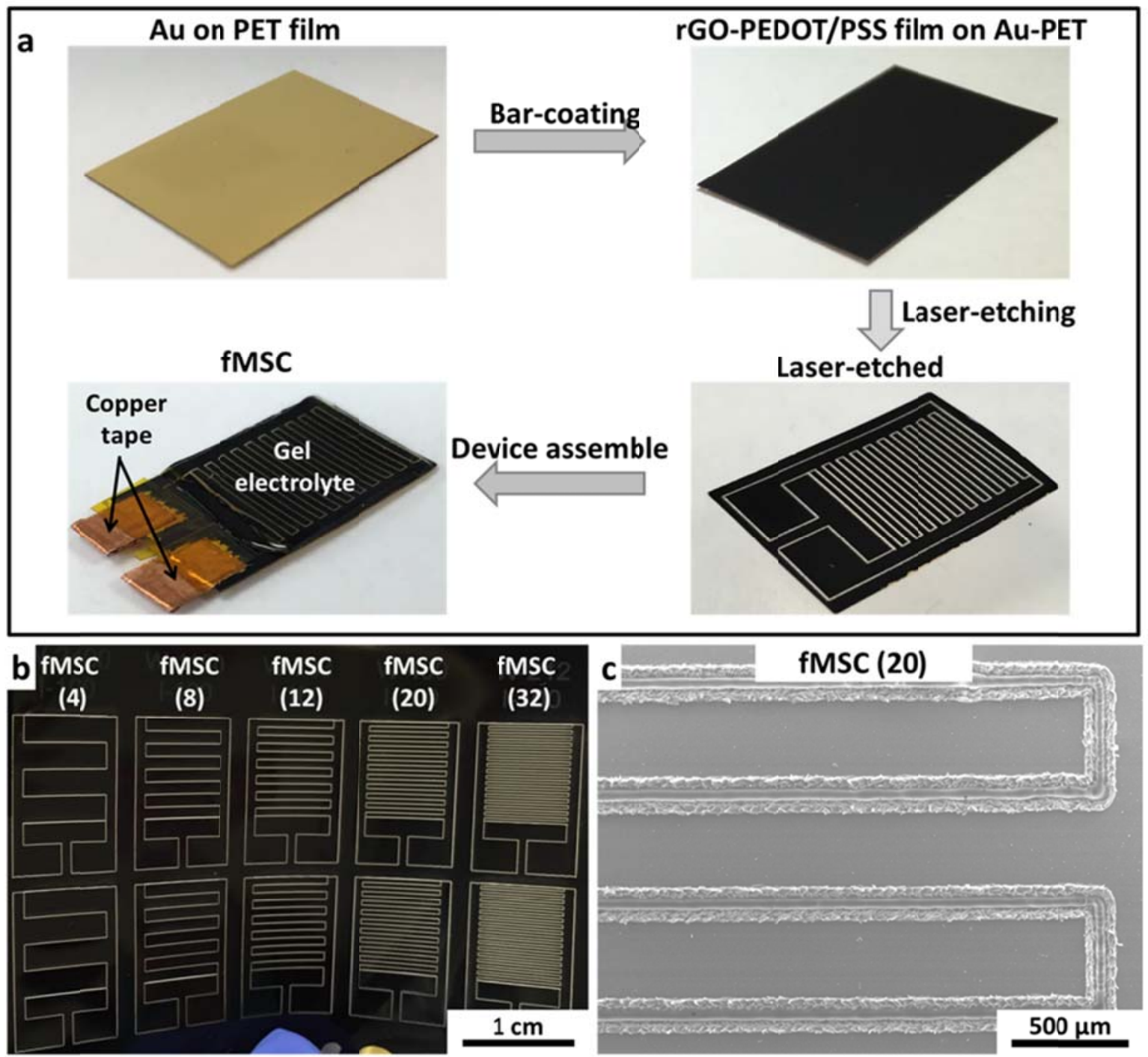

2 Figure 1. (a) Schematic diagram of the fabrication of all-solid-state rGO-PEDOT/PSS based

3 planar fMSCs on a flexible PET film using laser cutter technique. (b) Photo images of laser-

4 etched fMSCs with different configurations on one rGO-PEDOT/PSS film. (c) SEM image of

5 fMSC (20) pattern (top view). 

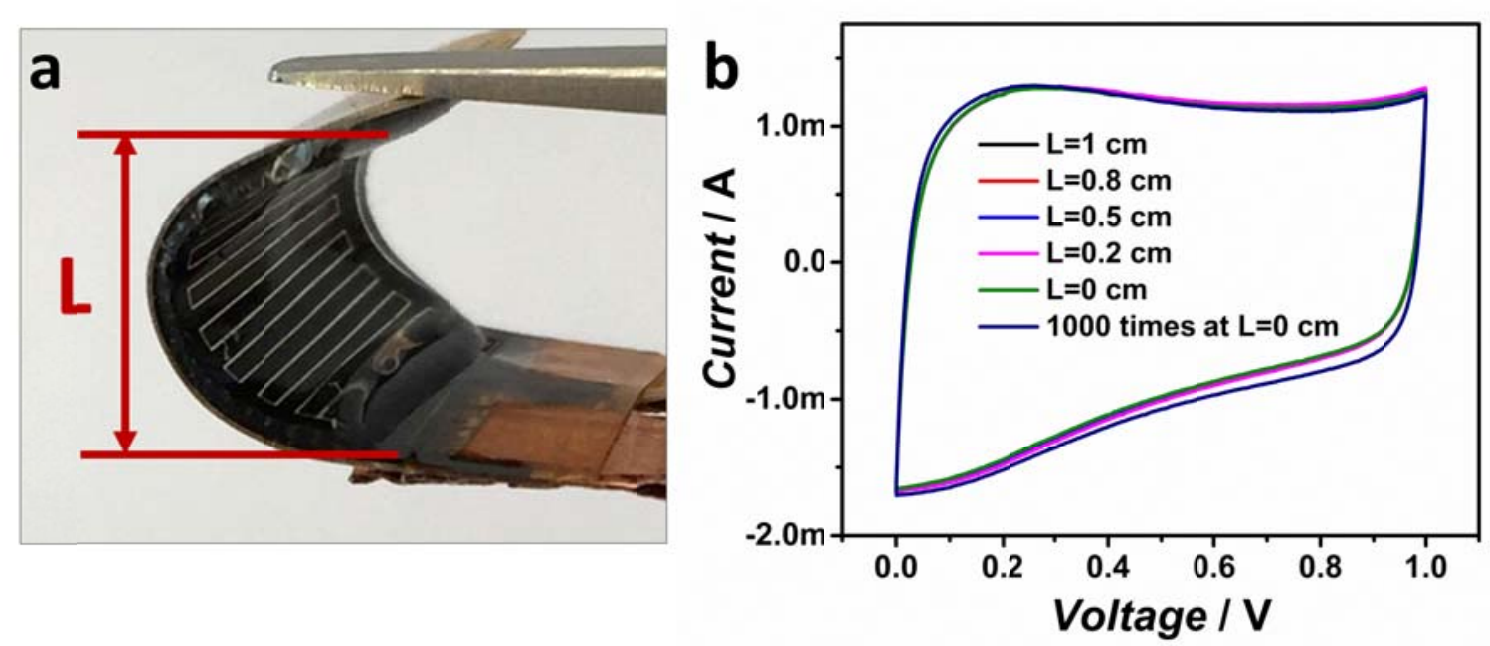

2 Figure 2. (a) Photograph of assembled fMSC device on its bent state. (b) Comparison CVs of

3 the $\mathrm{fMSC}$ device before $(\mathrm{L}=1 \mathrm{~cm})$ and after bending at different curvatures $(\mathrm{L}=0.8,0.5,0.2$,

4 and $0 \mathrm{~cm}$ ). Also shown is the $\mathrm{CV}$ after repeatedly bending the $\mathrm{fMSC}$ device from $\mathrm{L}=1$ to $\mathrm{L}=0$

5 for 1,000 times. The scan rate used is $50 \mathrm{mV} \mathrm{s}^{-1}$.
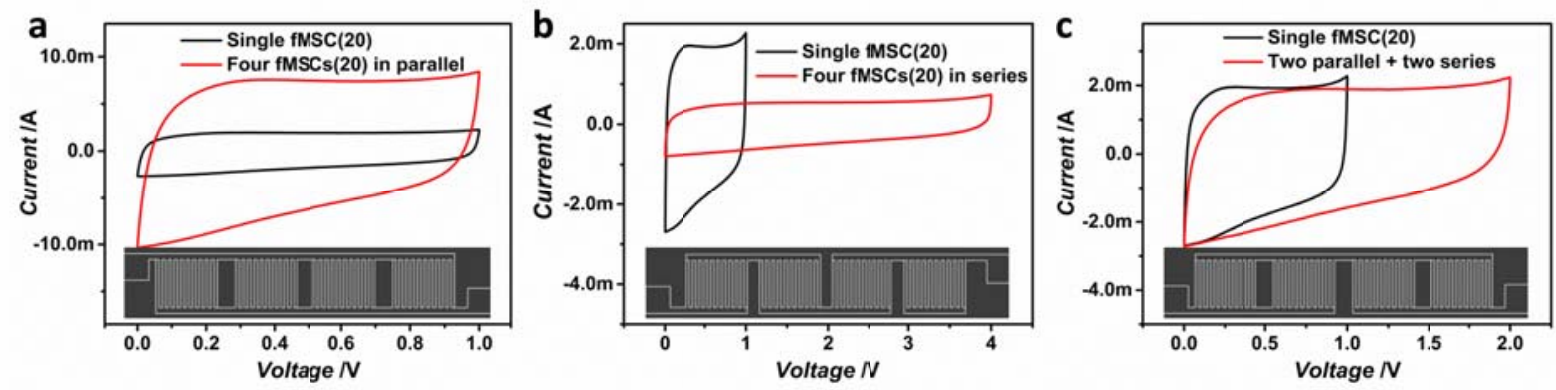

7 Figure 3. $\mathrm{CVs}$ (scan rate $=100 \mathrm{mV} \mathrm{s}^{-1}$ ) for four micro-supercapacitors connected (a) in parallel,

8 (b) in series, and (c) in a combination of series and parallel. 

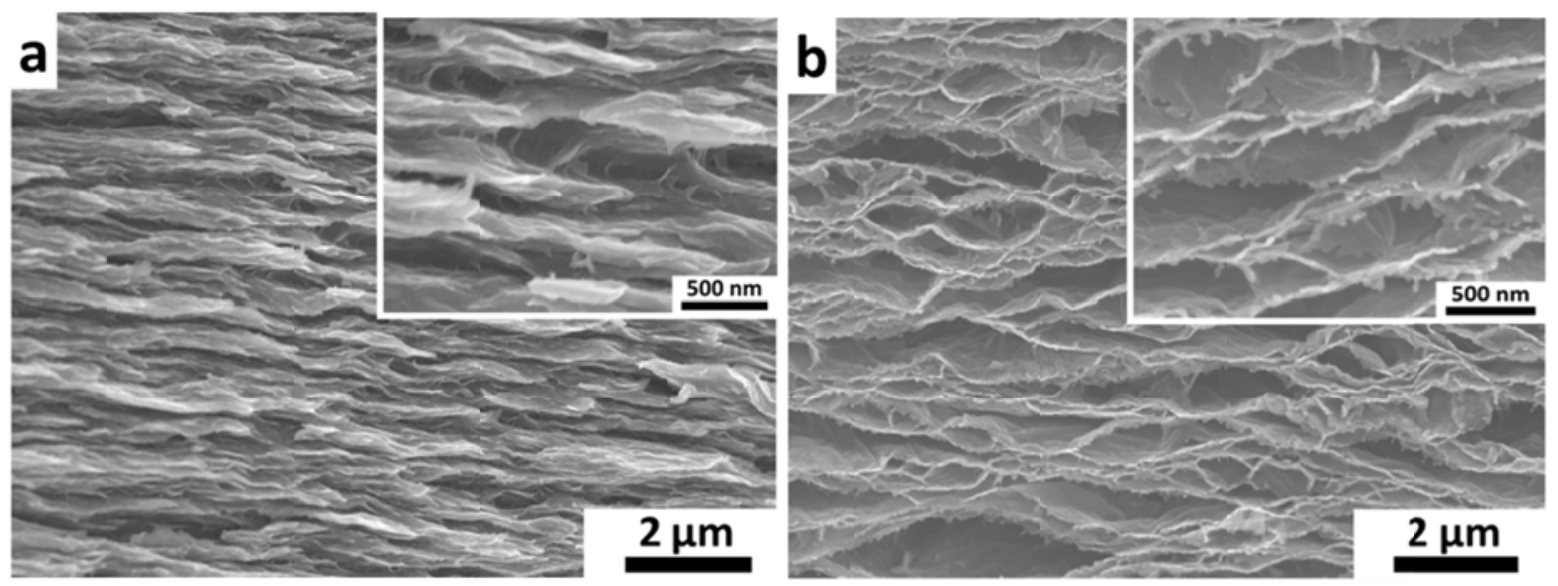

Figure 4. (a) Cross-section SEM images of (a) rGO-PEDOT/PSS parent film (non-laser-cut)

3 and (b) laser-cut rGO-PEDOT/PSS film.
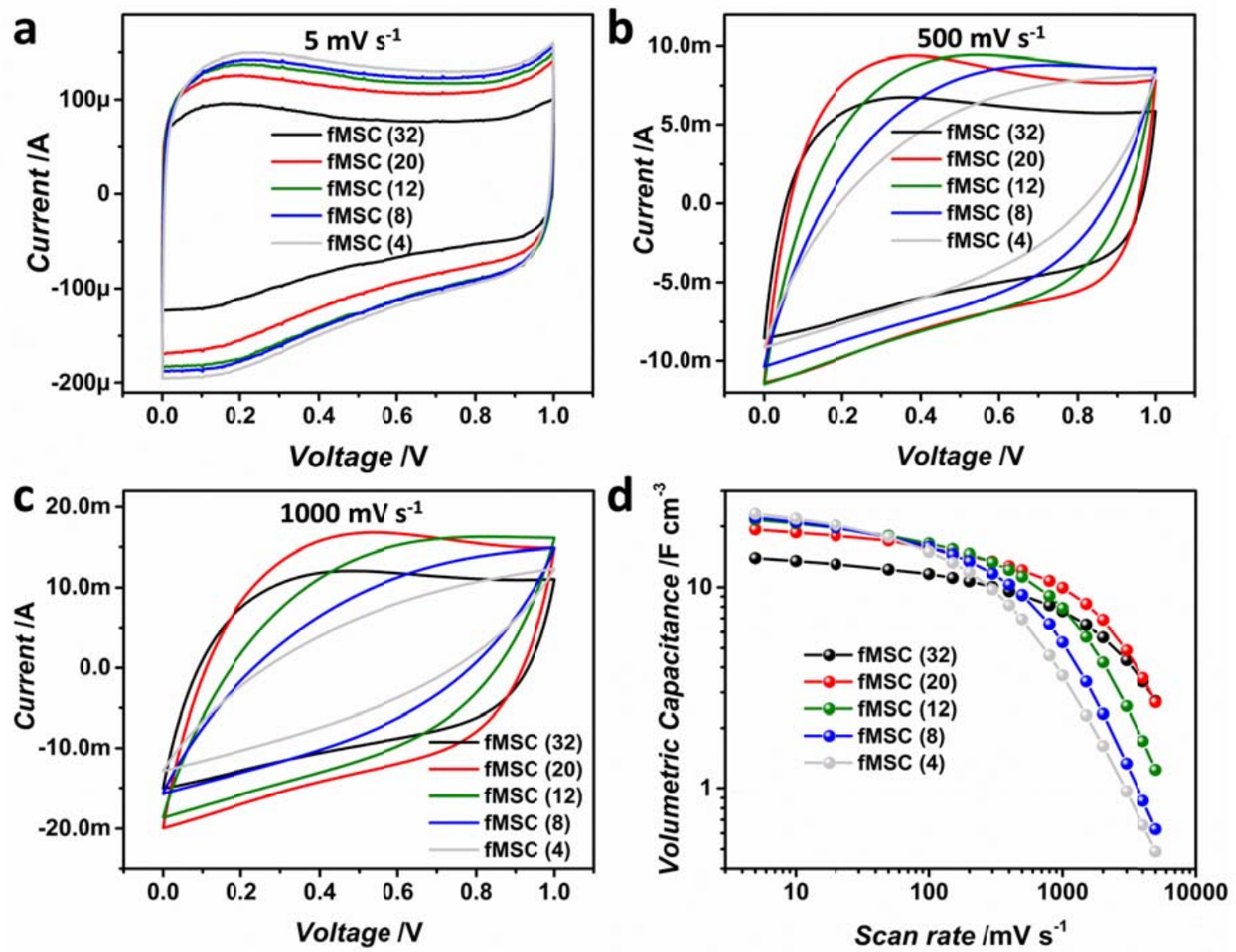

5 Figure 5. Dependence of the electrochemical properties of fMSCs on width of microelectrode

6 fingers. (a) $\mathrm{CV}$ curves at $5 \mathrm{mV} \mathrm{s}^{-1}$ (b) $\mathrm{CV}$ curves at $500 \mathrm{mV} \mathrm{s}^{-1}$. (c) $\mathrm{CV}$ curves at $1000 \mathrm{mV} \mathrm{s}^{-1}$.

7 (d) Volumetric Capacitance calculated from CVs. 

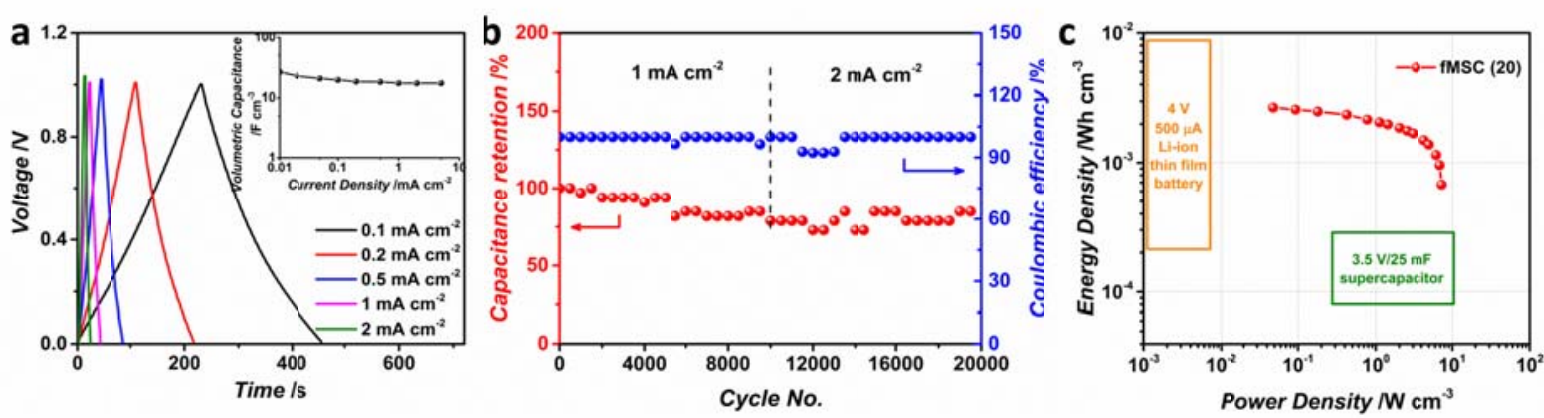

2 Figure 6. Electrochemical performance of fMSCs (20). (a) Galvanostatic charge/discharge

3 curves at different current densities (with inset capacitance vs. current density).

4 Capacitance retention and coulombic efficiency during the 20,000 cycles. (c) Ragone plot of

$5 \quad \mathrm{fMSC}(20)$ and commercially available electrochemical devices.
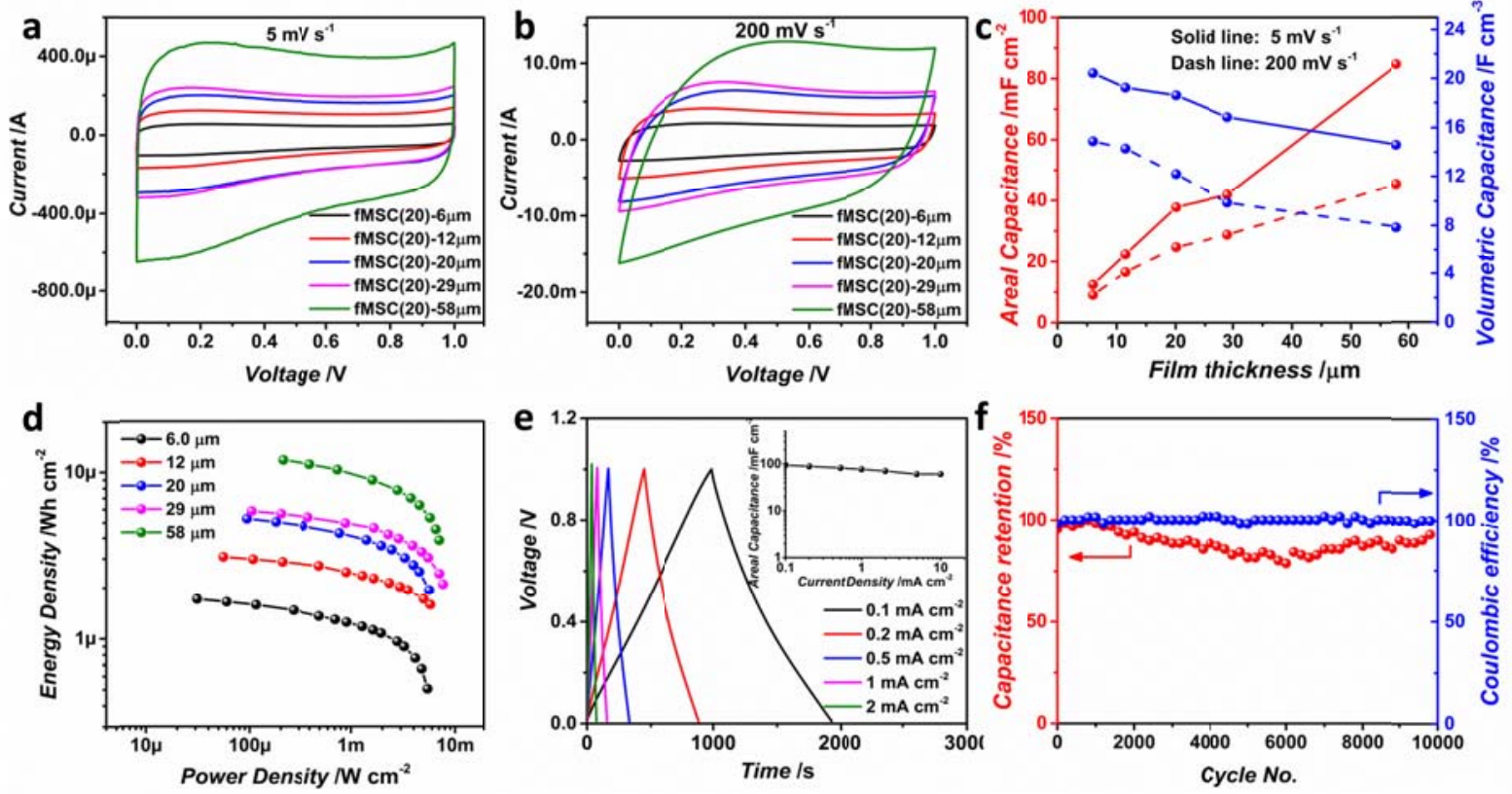

7 Figure 7. Dependence of the electrochemical properties of fMSCs on electrode thickness.

8 (a) $\mathrm{CV}$ curves at $5 \mathrm{mV} \mathrm{s}^{-1}$ (b) $\mathrm{CV}$ curves at $200 \mathrm{mV} \mathrm{s}^{-1}$. (c) Areal and volumetric capacitance

9 vs. thickness at a scan rate of $5 \mathrm{mV} \mathrm{s}^{-1}$ (solid line) and $200 \mathrm{mV} \mathrm{s}^{-1}$ (dash line). (d) Ragone

10 plots. (e) Galvanostatic charge/discharge curves of $\mathrm{fMSC}(20)-58 \mu \mathrm{m}$ at different current

11 densities (with inset capacitance vs current density). (f) Capacitance retention and coulombic

12 efficiency of $\mathrm{fMSC}(20)-58 \mu \mathrm{m}$ during the 10,000 cycles. 


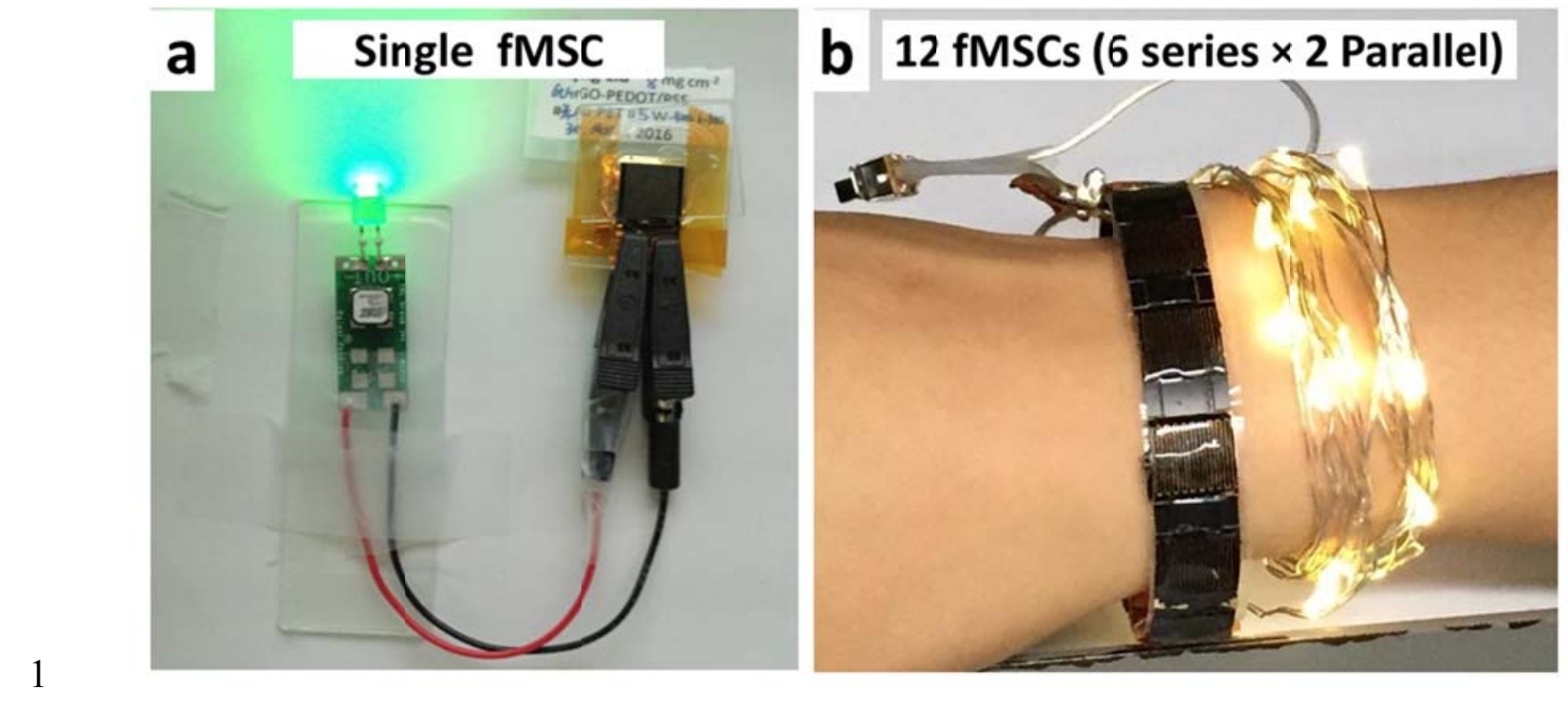

2 Figure 8. Optical images of (a) a green light-emitting diode (LED) powered by single $3 \mathrm{fMSC}(20)-58 \mu \mathrm{m}$ (charged to $1 \mathrm{~V}$ ); (b) 20 LEDs powered by $12 \mathrm{fMSCs}$ connected in 6 series $4 \times 2$ parallel (charged to $6 \mathrm{~V})$ on a long strip of flexible $\mathrm{rGO}$-PEDOT/PSS film $(1.5 \mathrm{~cm} \times 18$ $5 \mathrm{~cm}$ ). 
Flexible microsupercapacitors (fMSCs) with different pattern designs were facilely fabricated via laser-etching on flexible rGO-PEDOT/PSS films. Optimized fMSCs delivered high areal capacitance $\left(84.7 \mathrm{mF} \mathrm{cm}{ }^{-2}\right)$, volumetric capacitance $\left(14.5 \mathrm{~F} \mathrm{~cm}^{-3}\right)$ and high cycling stability (94.3\%, 10,000 cycles). 12 tandem connected fMSCs can power 20 light-emitting diodes even when bent demonstrating versatility for practical applications.

Keyword: micro-supercapacitor, laser-etching, flexible, high energy density, graphene 8

Yuqing Liu ${ }^{1}$, Bo Weng ${ }^{2}$, Qun Xu ${ }^{3}$, Yuyang Hou ${ }^{1}$, Chen Zhao ${ }^{1,4}$, Stephen Beirne ${ }^{1}$, Kewei Shu ${ }^{1}$, Rouhollah Jalili ${ }^{1}$, Gordon G. Wallace ${ }^{1}$, Joselito M. Razal ${ }^{5, *}$, and Jun Chen ${ }^{1, *}$

\section{Facile Fabrication of Flexible Micro-Supercapacitor with High Energy Density}

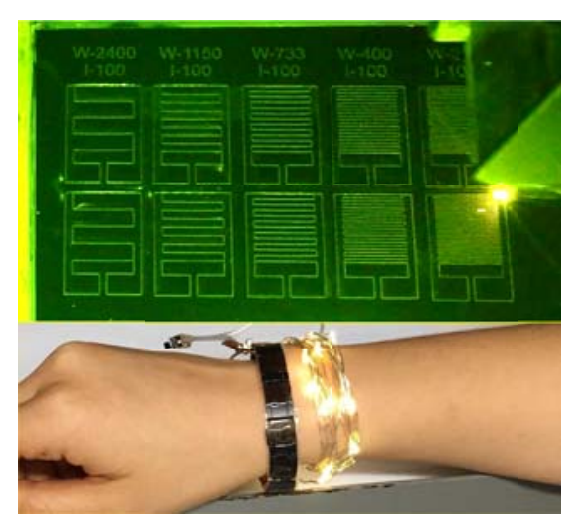

